\title{
THE ICONICITY OF PICTURE COMMUNICATION SYMBOLS FOR RURAL ZULU CHILDREN
}

\author{
Lize Haupt and Erna Alant*
}

Centre for Augmentative and Alternative Communication

University of Pretoria

\begin{abstract}
The purpose of this study was to investigate the iconicity of selected Picture Communication Symbols (PCS) for rural Zulu ten-year-olds. Participants were presented with copies of a commercially available communication overlay without glosses. They were required to match a symbol with each of 36 spoken Zulu labels. With both strict and lenient scoring criteria applied, $2.8 \%$ and $11.1 \%$ (respectively) of the symbols on the communication overlay emerged as iconic for participants. It was further established that the position of symbols on the overlay, the total frequency of selection of symbols, and gender did not influence results. An analysis of errors revealed that for some symbols many of the participants agreed on a single specific label, be it the target label or a non-target label; while for other symbols there were either many possible labels, or none. The term distinctiveness was coined to describe how well defined or specific were the evoked meanings triggered by a symbol in the viewers' minds. Results suggest that participants did not make maximum use of the information provided by arrows in the symbols. This finding could be ascribed to the opaqueness of arrows and participants' lack of previous experience with these conventional cues in pictures, as well as the traditional oral nature of the Zulu culture.
\end{abstract}

Key words: Augmentative and alternative communication (AAC), communication overlay, cross-cultural, iconicity, zulu, picture communication symbols (PCS), translation

\section{INTRODUCTION}

It is often stated that good practice when first teaching symbols, is to select symbols that are easy to learn (Fuller, 1997; Lloyd \& Fuller, 1990; Mirenda \& Locke, 1989). This strategy facilitates communication while at the same time ensuring success, which motivates the user. Iconicity information can greatly aid clinicians in such a selection, since iconic symbols are easier to learn (Fuller, 1987; Fuller, 1997; Lloyd \& Fuller, 1990; Lloyd, Fuller \& Arvidson, 1997; Lloyd, Loeding \& Doherty, 1985; Luftig, 1983; Luftig, Page \& Lloyd, 1983; Mizuko, 1987). Furthermore, information about the iconicity of symbols is especially valuable in South Africa because of widespread illiteracy. A literate communication partner can read the gloss (written text) that accompanies a symbol, but illiterate partners have to rely on the iconicity of symbols to guess their meaning. It is expensive and virtually impossible to train all possible communication partners in the use of the relevant symbol set/system, therefore the use of iconic symbols is more efficient.

Iconicity is defined as the degree to which an individual perceives visual similarity between a symbol and its referent (Blischak, Lloyd \& Fuller, 1997). Factors influencing visual perception would probably influence a symbol's iconicity for a given viewer. Such factors include the material on which symbols are printed (Deregowski, 1980a,b); schooling (Duncan, Gourlay \& Hudson, 1973); thinking styles (Retief, 1988; Taylor \& Clarke, 1994; Witkin, 1967); oral or literate background of viewer (Ong, 1982) and previous experience with symbols (DeLoache, 1991). Closer inspection of these factors reveals that they are all intertwined with culture. Culture is 'a set of behaviours, institutions, beliefs, technologies and values invented and passed on by a group of individuals to sustain what they believe to be a high quality of life and to negotiate their environments' (Taylor \& Clarke, 1994, p:-103).

It therefore seems reasonable to assert that iconicity shoúld be studied in the context of a culture, and that it cannot be taken for granted that results obtained from studying one group of people can necessarily be generalised to another. However, the iconicity of graphic representational systems has not previously been investigated in the context of any of South Africa's many cultures. There is a need for culture-specific iconicity information in this country to enhance alternative and augmentative communication (AAC) intervention for individuals with little or no functional speech (LNFS).

KwaZulu-Natal is the province in South Africa with the second highest disability prevalence rate $(6.7 \%)$ (Schneider et al., 1999). Furthermore, the 1996 census showed that $22.9 \%$ of South Africans are Zulu mothertongue speakers (Burger, 2000), making it one of the largest linguistic groupings in the country. Picture Communication Symbols (Johnson, 1981, 1985, 1992) is a set of aided, static communication symbols and is regarded as relatively iconic compared to other aided symbol sets and systems (Mirenda \& Locke, 1989; Mizuko, 1987). Although it originated in the USA, it is widely used all over the world, including South Africa. An investigation into how Zulu children relate to Picture Communication Symbols (PCS) could yield valuable information on how to modify the content, appearance or use of PCS to facilitate symbol learning and use.

A review of iconicity literature revealed that one of two methodologies is normally used to determine the iconicity of symbols: either the participant is shown a The South African Journal of Communication Disorders, Vol. 49, 2002 
symbol and asked to guess its meaning, or participants are required to match a spoken label with a symbol from a set of closed alternatives. It has been reasoned that such a forced-choice task might be easier than an openchoice task (Musselwhite \& Ruscello, 1984), probably resulting in the best possible iconicity values.

In a critique of their own study, Mirenda and Locke (1989) mentioned that communication overlays typically contain more than two symbols. They maintained that the inclusion of a larger number of symbols in iconicity tasks might yield more accurate results for intervention, purposes. In the light of this consideration it was decided to investigate iconicity in the context of a communication overlay. Instead of presenting a participant with three to five symbols to choose from, an entire overlay was presented. The communication overlays designed by Goossens', Crain and Elder (1996) can be photocopied directly from the manual, and are widely used unmodified in South Africa. The use of a 36-matrix overlay from this collection therefore seemed appropriate for the present study.

Clearly such a task would differ from those in previous iconicity studies in four important ways. Firstly, when participants are presented with a complete communication overlay, the set of alternatives is substantially larger than in previous studies, as has just been discussed. Secondly, all symbols are semantically related to the same theme and therefore possibly to each other, even if indirectly. Thirdly, the 36 symbols comprising the set of, alternatives will remain static across all 36 trials; and fourthly, each symbol will in time be the target symbol. These last two factors create the possibility that some participants may remember which symbols they had chosen for several consecutive trials and, in response to the next labels, narrow their selection down to those not yet chosen. The possibility that a combination of these factors might influence the iconicity values obtained should be kept in mind.

Due to the novel methodology, the question arosie as to what terminology should be used. Transparency refers to the ease of identification of symbols when no additional cues, such as printed labels or verbal hints, are provided' (Musselwhite \& Ruscello, 1984, p.437). In this study, however, an additional cue was provided in that the theme of the overlay was known. Thus the traditional term 'transparency' could not be used. Translucency on! the, other hand (typically determined by participants rating on a 5- or 7-point scale) indicates the extent to which a symbol looks like its referent (Blischak et al., 1997). No ratings were required from participants in the present study, so the term translucency would not apply either. It was decided that use of the more general term iconicity would be most accurate.

\section{METHOD}

\section{Aim}

The primary aim of the study was to determine how accurately typically developing rural Zulu 10-yearolds could identify 36 Picture Communication Symbols (PCS), presented thematically on a commercially available communication overlay, in response to spoken labels. The following objectives were formulated: to select a commercially available communication overlay, which contained no concepts that would be foreign to rural Zulu children; to determine how accurately tenyear-old Zulu children would select the correct symbol in response to its spoken label; to describe error patterns; and to investigate factors that could have influenced results, specifically total frequency of selection, position on overlay and gender

\section{Research design}

The nature of the study was exploratory. An analytical survey was conducted in which 94 rural Zulu mother-tongue speakers from nine schools were exposed to $36 \mathrm{PCS}$ symbols in the context of a commercially available communication overlay. In response to a verbal Zulu label they had to mark the symbol they thought best depicted that concept. Sampling was purposive in the sense that schools were selected according to accessibility. At the selected schools however, all children that met the selection criteria were included in the study. The data was quantitative in nature and was therefore subjected to statistical analysis to obtain iconicity values. Possible influences on results and error patterns were analysed qualitatively.

\section{PARTICIPANTS}

\section{Participant selection criteria}

Participants had to be Zulu mother-tongue speakers between the ages of 10 and 11 , with no indication of hearing loss or uncorrected sight problems. They had to be in either grade 4 or grade 5, to ensure comparable educational and experiential backgrounds; and should never before have failed a school year. In the absence of formal assessment of mental abilities, this criterion was included to control for severe learning and mental disabilities.

\section{Description of schools}

The KwaZulu-Natal Department of Education and Culture divides the province into 196 circuits. The twenty-two primary schools in the Kranskop East Circuit were targeted for this study. These schools are all located along three main routes, and three schools along each route, that would be accessible by sedan vehicle, were chosen in consultation with a physical planner from the KwaZulu-Natal Department of Education and Culture Pietermaritzburg region. Permission to perform the study was obtained from the principal of each selected school, as well as the KwaZulu-Natal Department of Education and Culture.

The nine schools that were selected were all co-educational government-funded schools. None were boarding schools, which means that all the participants were indigenous to the Kranskop area. None of the schools had facilities for learners with special educational needs. Although the mother tongue of all participants was Zulu, the official language of instruction at all schools was English. The KwaZulu-Natal Department of Education and Culture classifies schools as deep rural, rural, peri-urban and urban, according to no set definitions. Rural is simply described as far from any town, whereas deep rural means 'off the beaten track' ( $P$. Müller, Deputy Chief Education Specialist in Education Management Information Services, personal communication, July 9, 2001). All schools in the sample 
were classified as rural or deep rural by the KwaZuluNatal Department of Education and Culture.

\section{Description of participants}

At the nine selected schools all children who met the selection criteria were identified. A population of 94 children were tested, of which 52 were female and 42 male. Participants were between the ages of 10 and 11 , and the mean chronological age was ten years and five months.

\section{PROCEDURE}

\section{Preparatory phase}

This phase included the selection and translation of a suitable communication overlay (Goossens' et al., 1996), development of a test protocol, training of the research assistant and execution of a pilot study to pretest the validity of the translation and test protocol.

The aim of the selection process was to minimise cultural distance between the concepts represented on the overlay and the experiential background of the target population. The basic content of the overlay had to form part of the world knowledge of children from the target population. The researcher was assisted in the selection process by a panel of three Zulu judges each of whom had between four and 15 years' teaching experience in the Kranskop area.

To minimise the influence of linguistic factors on the performance of participants, the entire procedure was conducted in Zulu. Consequently all labels (the sentences/phrases accompanying the symbols on the overlay) had to be translated into Zulu. The goal was to produce an ethnographic translation (Brislin, 1980) and a process consisting of blind back-translation, a review committee and pre-test procedures (Bracken \& Barona, 1991; Retief, 1988) was followed. The translation process involved eight people, five of whom were Zulu mother-tongue speakers with proficiency in English; and three of whom were English mother-tongue speakers with proficiency in Zulu. All eight translators had previous experience with translation between the two languages. The final translations are presented in Table 1

A young Zulu. adult served as research assistant. She had obtained her Senior Certificate and was at the time studying part time through Unisa. Additionally, she co-reared eight younger cousins and did PRO work for two youth organizations in the Kranskop area. She was selected for her proficiency in English and good rapport with young children.

As an introduction to the training, the research assistant received general background information on the aims of the study, and was presented with the communication overlay as well as the Zulu and English phrases. Thereafter the procedure was performed once on each of three children who met the selection criteria, but who were not included in the pilot or main studies: These sessions were performed as part of training, and in order to develop a practical test protocol. During the first session the research assistant worked from a crude protocol designed by the researcher, and instructions were modified in consultation with the researcher where it seemed necessary. During sessions two and three the protocol did not change considerably, and the research assistant reported familiarity with the procedure.

A pilot study was then performed in two phases. During the first phase the procedure was performed on ten participants who met the selection criteria, but who were not included in the main study. Based on the results of this phase, minor adjustments were made to the protocol and translation. Three modelled training items and three independent training items proved to be sufficient to train participants in the task, but it seemed necessary to add an instruction to scan through all the symbols before making a choicc. Such an instruction was added to the protocol. Participants reported that the translation. for the phrase 'Let us ' put " on...' ('Masendlale...') was too close to the translation for 'Let us make the bed' ('Asendlale umbhede'). Consequently an alternative translation ('Maseleke...'), that satisfied all the participants as well as the translators, was used.in the main study.

The second phase of the pilot study entailed performing the procedure a second time on the same participants, one week after the first administration. Results were compared in order to establish test-retest reliability.

\section{Table 1: Final translations of labels}

\begin{tabular}{|c|c|}
\hline English phrase & Zulu translation \\
\hline What a mess! & Kwaze kwangcola! \\
\hline It looks like a bòmb went off! & Sengathi kuqhume ibhomu! \\
\hline It is dirty. & Kungcolile. \\
\hline You need to change them. & Udinga ukuwàshintsha. \\
\hline Let us take it off. & Asikususe. \\
\hline Help me, please. & Ngicela ungisize. \\
\hline It is finished. & Kuphelile. \\
\hline What is next? & Kulandelani? \\
\hline Put it in the tub. & Faka kubhavu. \\
\hline Let us make the bed. & Asendlale umbhede. \\
\hline Hold this, please. & Ngicela ubambe lokhu. \\
\hline You need to pull. & Udinga ukudonsa. \\
\hline It is crooked. & Kugwegwile. \\
\hline Let us do it again. & Asiphinde futhi. \\
\hline Fold it back. & Kugoqele emuva. \\
\hline Tuck it in. & Kushutheke. \\
\hline Let us put on... & Maseleke... \\
\hline ...the sheets & ... amashidi. \\
\hline ...the blanket & ...ingubo. \\
\hline ...the pillowcase & ...iphilo. \\
\hline Thank you. & Ngiyabonga. \\
\hline You are welcome. & Wamukelekile. \\
\hline Let me:... . & Ake ngi... \\
\hline Where is it? & Kuphi? \\
\hline Put it here. & Beka lapha. \\
\hline Puff it up. & Khukhumalisa. \\
\hline It is nice and soft. & Kuntofontofo. \\
\hline What do you think? & Ubona kanjani? \\
\hline It looks good. & Kubukeka kahle. \\
\hline It looks bad. & Kubukeka kabi: \\
\hline Whoops! & We! \\
\hline Look at this. & Buka lokhu. \\
\hline We forgot. & Sikhóhliwe. \\
\hline Yes. & Yebo. \\
\hline No. & Cha. \\
\hline It is nice and clean. & Kuhlanzeke kahle. \\
\hline
\end{tabular}

The South African Journal of Communication Disorders, Vol. 49, 2002 


\section{MAIN STUDY}

The researcher and research assistant visited the nine schools and included all children that met selection criteria at every school. Sessions were conducted with ten or less participants at a time (except in two cases where twelve children were included due to time constraints) and took between 45 minutes and one hour. Each participant was issued with a recording booklet (containing 36 A4 facsimiles of the communication overlay and six facsimiles of the training overlay) and a felt tip marker. The training procedure consisted of six trials where participants were asked to indicate the written word on the training overlay that corresponded with the Zulu word that the research assistant read out. Participants were shown and told to visually scan the entire matrix before indicating the word. Since children who experience problems with sight or hearing would have difficulty with this task, it served simultaneously as hearing and visual screening. During the testing procedure, Zulu labels were read in an order that was determined before commencement of the study by drawing symbols randomly from a bag and assigning each number accordingly. Participants had to mark one label per page. Every label was repeated once and the procedure was not timed. After the session each participant received a token. Teachers were given information on AAC in general and the aims of the study, as well as worksheets for their classes. They were asked

Table 2: Presentation order of symbols

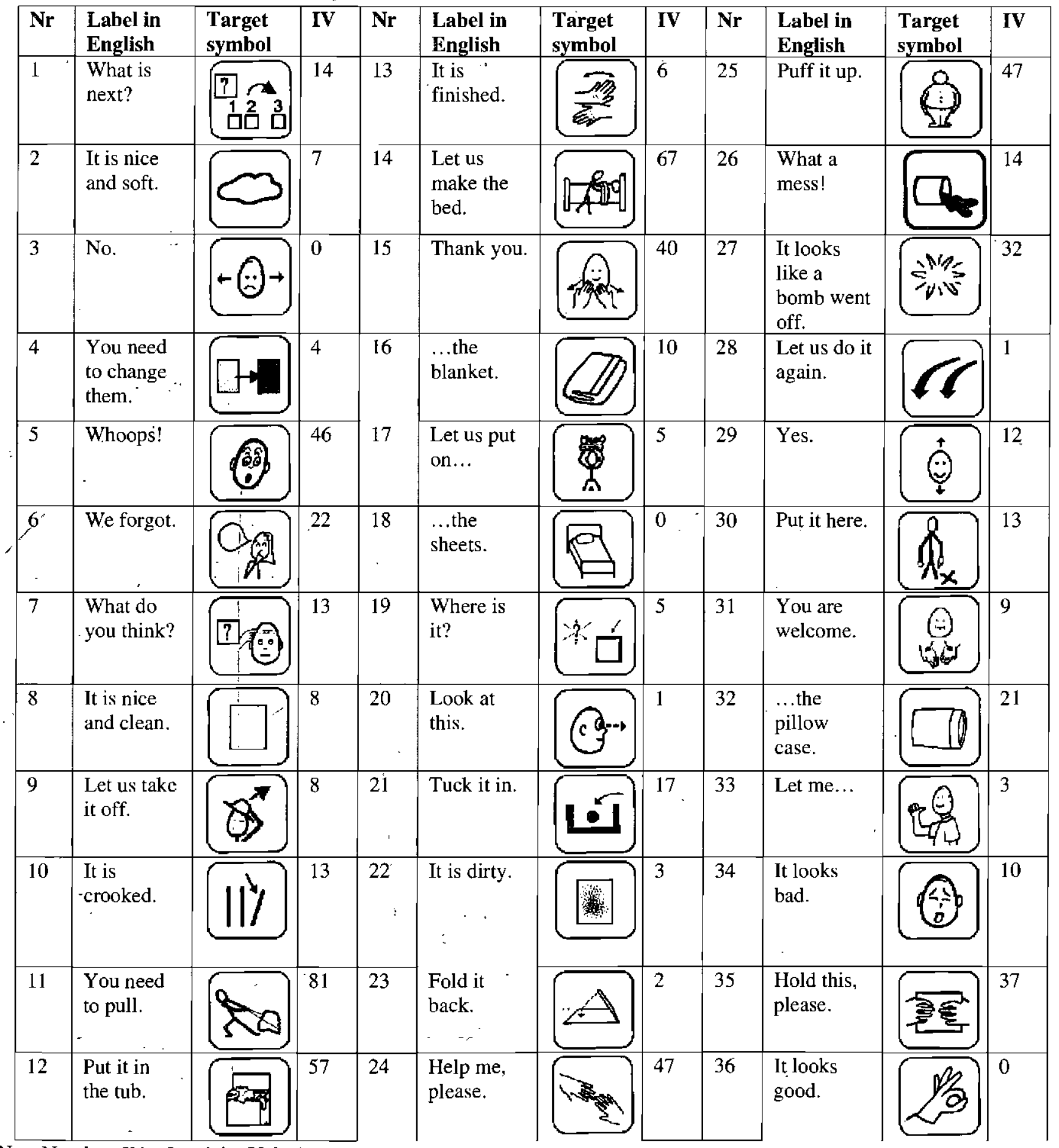


to discuss the session with their pupils with the help of the worksheets, as a form of debriefing.

\section{Data analysis}

Paired t-tests were performed on the two sets of data from the pilot study in order to reveal possible significant differences between the performances of the same group of participants one week apart. The performance of males and females in the main study were compared in a chi-square test. Descriptive statistics, including - frequency distribution counts, mean and standard deviation were also used. Possible influences on results and error patterns were analysed qualitatively.

\section{RESULTS AND DISCUSSION}

The results are described and discussed according to the objectives of this study. The frequency of correct responses per label is presented. An analysis of errors is presented and discussed, and possible influences on correct responses are considered.

Frequency of correct responses per label

\section{Missing data}

Out of the total of 3,384 responses generated in this study (94 participants x 36 trials), two symbols on one page were marked on five occasions, no choice was indicated on eight occasions, and the activity graphic in the top right hand corner of the overlay was chosen on nine occasions. In order to focus on the most salient patterns in this study, it was arbitrarily decided to consider only those responses that were indicated by at least $20 \%$ of the participants (i.e. 19 participants or more). Consequently the influence of the missing data was deemed negligible.

\section{Iconicity values}

To aid in interpretation of the results, the number as well as iconicity value of each symbol are presented in Table 2 . The iconicity value represents the number of participants that chose a symbol in response to its target label.

Doherty, Daniloff \& Lloyd (1985) used strict (iconicity values $\geq 75 \%$ ) and lenient (iconicity values $\geq$ $50 \%$ ) criteria for interpreting the transparency scores of Amer-Ind gestures. Although the present study did not investigate pure transparency, these criteria were nevertheless deemed useful. In accordance with the above criteria, one symbol (symbol 11) was found to be iconic when the strict criterion was applied, and four symbols (symbols $11 ; 12 ; 14$; and 25 ) when the lenient criterion was applied. Note that symbol 5 achieved an iconicity value of $49 \% \quad(n=46)$ and was one response short of being classified as iconic according to the lenient criterion. Thus either $2.8 \%$ or $11.1 \%$ of the symbols on the communication overlay were iconic for the participants involved, depending on the criterion used. The average of 'correct' responses across all symbols was 17.75 $(18.88 \%)$ with a standard deviation of 20.17 . Analysis of errors
When the highest frequency responses were studied for each symbol, it became clear that for some symbols many participants agreed on a single specific label, be it the target label or a non-target label. For other symbols either many possible labels, or none of the labels, were indicated. The term distinctiveness was coined to describe how well defined or specific were the evoked meanings triggered by a symbol in the mind of a viewer. This term should not be confused with 'perceptual distinctness', as described by Fuller, Lloyd, and Stratton (1997), which refers to the degree to which the symbols in a group are clearly different or distinct from one another.

It is also important to note that 'distinctiveness' was not intended as an. equivalent to 'iconicity'. Whereas both terms concern the visual relationship between a symbol and its referent, they indicate different aspects of that relationship. Iconicity pertains to the degree of visual similarity perceived, as demonstrated by the use of the three dimensions, transparency, translucency and opaqueness (Blischak et al., 1997). The term distinctiveness, as used in this study, relates to the specificity of visual similarity perceived i.e. whether participants perceive similarity to one referent, to many, or to none.

Since iconicity and distinctiveness are not opposing terms, a symbol can be classified by both terms simultaneously. Such a classification will lead to all symbols falling into one of four orthogonal groups: distinctive and more iconic (many participants chose a certain symbol in response to its target label only); indistinctive and more iconic (many, participants chose a certain symbol in response to its target label, however they also chose that symbol often in response to one or more other labels); distinctive and less iconic (few participants chose a certain symbol in response to its target label, however many of them chose that symbol in response to a certain non-target label); indistinctive and less iconic (few participants chose a certain symbol in response to its target label but they chose that symbol often in response to one or more other labels).

The symbols from the present study were distributed across these four orthogonal groups. To determine iconicity and distinctiveness two criteria were used. Regarding the iconicity of a symbol, the criterion suggested by Hoemann (1975) was used (iconicity values $\geq 25 \%$ ). Admittedly this criterion is very lenient (Lloyd et al., 1985), but since this analysis was concerned with relative rather than absolute iconicity, it was considered appropriate. To describe distinctiveness, all response frequencies $\geq 20 \%$ were investigated. Note that this cut-

Table 3: Distribution of symbols according to iconicity and distinctiveness

\begin{tabular}{|c|c|c|}
\hline $\begin{array}{c}\cdots \\
\ldots \\
\end{array}$ & $\begin{array}{l}\text { More iconic } \\
\text { (iconicity values } \geq \\
25 \% \text { ) }\end{array}$ & $\begin{array}{l}\text { Less iconic (iconicity } \\
\text { values }<25 \% \text { ) }\end{array}$ \\
\hline $\begin{array}{l}\text { Distinctive (only one } \\
\text { response over } 20 \% \text { ) }\end{array}$ & $\begin{array}{l}\text { Numbers: } 5 ; 12 ; 16 ; \\
25 ; 27 ; 35\end{array}$ & $\begin{array}{l}\text { Numbers: } 4 ; 7 ; 13 ; 15 ; 20 \\
26 ; 28 ; 31\end{array}$ \\
\hline $\begin{array}{l}\text { Indistinctive (More than } \\
\text { one response over } 20 \% \text {, } \\
\text { or no responses over } \\
20 \% \text { ) }\end{array}$ & Numbers: $11 ; 14$ & $\begin{array}{l}\text { Numbers: } 6 ; 18 ; 32 ; 33 ; 1 ; \\
2 ; 3 ; 8 ; 9 ; 10 ; 17 ; 19 ; 21 ; \\
22 ; 23 ; 24 ; 29 ; 30 ; 34 ; 36\end{array}$ \\
\hline
\end{tabular}


off point was arbitrarily selected and not statistically determined. The distribution of the symbols from the present study across these four orthogonal classifications is presented in Table 3.

Although the variable 'distinctiveness' has never been investigated before, it was hypothesised that it would yield valuable information as to how participants viewed symbols. Hence a discussion of symbols according to this classification follows, including only the most salient points. For a detailed discussion the reader is referred to Haupt (2001).

\section{Distinctive and more iconic}

Six symbols $(5,12,16,25,27$, and 35$)$ were classified as distinctive and more iconic. It seems that participants perceived a relatively strong visual relationship between these six symbols and their target referents, and to those referents only. It is hypothesised that of all 36 symbols included in the study these six will probably be the easiest to learn for rural Zulu children.

\section{Indistinctive and more iconic}

Symbols 11 and 14 (the two symbols with the highest iconicity values) were classified as indistinctive and more iconic. It is evident that not only did participants perceive visual similarities between these symbols and their target referents, but also to other referents.

It can be argued that the conceptual features of symbol 14 were exceptionally close to the theme of the overlay (Making the bed). Underlying similarities possibly existed between the label for this symbol and those for other symbols, making it a popular choice. It is postulated that the indistinctiveness of symbol 14 can therefore be ascribed to the context in which it was presented. Had this symbol been presented in a group of unrelated foils like in most other iconicity studies, it probably would have scored higher on distinctiveness.

The perceptual features of symbol 11 ("You' neéd to pull') include a human figure in implied motion 'and an object linked to the figure by rope. One of the two non-target labels that were associated with this symbol by more than $20 \%$ of participants also referred to physical motion ('Let us take it off') and the selection of this symbol can therefore easily be explained.

The other non-target label associated with this symbol referred to more abstract motion: from present to future ('What is next?'). The target symbol for this label (symbol 1) shows this motion with an arrow. It seems that participants preferred the symbol that implied motion by postural cues, to the symbol that included an arrow, suggesting the possibility that participants did not interpret the arrow as presenting information about movement. This phenomenon recurred throughout the analysis of error patterns and possible, causes are discussed later in the paper.

Another aspect to be considered is the fact that the label 'What is next?' is a question. Three of the 36 symbols on the overlay contained question marks: symbols 1, 7 and 19; whereas symbol 11 did not. It is postulated that if participants recognised and optimally utilised the question mark, they would have associated one of the three 'question mark symbols' with the label 'What comes next?'. The fact that they preferred symbol
11 may indicate that they did not interpret the question mark as indicating a question.

\section{Distinctive and less iconic}

Symbols 4, 7, 15, 28 and 31 were classified as distinctive and less iconic. Symbol 4 intends to depict a change of colour, however it could also be interpreted as a change from clean to dirty, which would account for the confusion with the label 'What a mess!'. If this explanation were accepted, it would appear that participants did interpret the arrow in this symbol as indicating change. It seems once again that the clue afforded by the question mark in symbol 7 was lost since participants associated it with a label that was not in question form.

To viewers that are unfamiliar with American Sign Language, symbols 13, 15 and 31 might be difficult to understand. Symbol 15 shows two hands on the chin of a face. The label 'We forgot' was associated with this symbol, possibly because people who are shocked or surprised sometimes put their hands over their mouths. If this interpretation is accepted, participants once again did not use information afforded by the arrows pointing outwards. Symbol 31 depicts two hands with empty palms turned upwards. If the hands were motionless in that position, it could be interpreted as showing that the hands are empty, possibly explaining why the label 'It is finished' was associated with this symbol. The arrows, however, imply movement away from the face, a clue that was presumably not interpreted as such by participants.

Symbol 28, depicted by two arrows pointing downwards, was associated with the label 'It is crooked'. The target label for symbol 28 is 'Let us do it again'. The participants, instead of perceiving that the arrows indicated repetition, perceived them as two crooked lines. This finding confirms the hypothesis that participants did not interpret arrows in the symbols as indicating direction or movement.

\section{Indistinctive and less iconic}

It is interesting to note that seven of the symbols in this classification (symbols $1,3,9,19,21,23,29$ ) contain arrows. The evidence collected thus far seems to suggest that the arrows could have been a cause of the low iconicity and indistinctiveness. For example, none of the symbols indicated by this study as iconic contained arrows, and only five symbols containing arrows were not classified as indistinctive and less iconic (symbols 4, $13,15,20,31$ ). It has however already been postulated that the arrows in symbols $13,15,20$ and 31 were not interpreted conventionally. Only in symbol 4 was the arrow interpreted as indicating change. Conversely it could be argued that in symbol 4 the form of the two objects stay the same and hence, unlike the other symbols containing arrows, only one attribute (colour) changes. The change is therefore highlighted, possibly contributing more to the target interpretation than the actual arrow. It seems that participants did not interpret arrows as indicating direction or change in any of the symbols except possibly one. Two possible explanations for this phenomenon will be discussed.

The arbitrary nature of arrows necessitates previous experience with it in order to be able to interpret it. It could be argued that children from rural areas are 


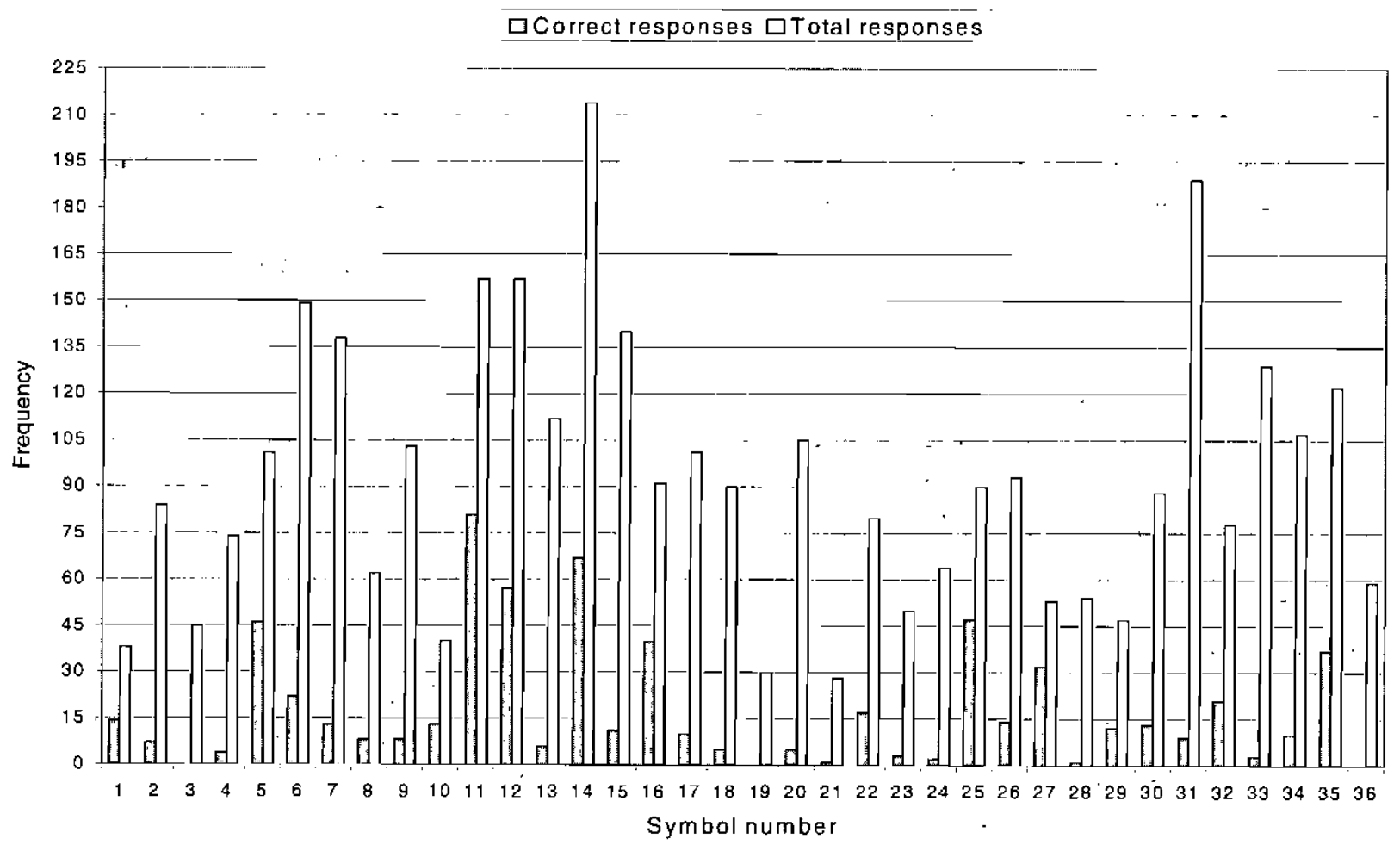

Figure I: Correct responses and total frequency of selection

not afforded enough opportunity to experience and learn to interpret such westem conventions. Yet the participants in this study all attended school where encounters with such conventions could be expected. It seems possible that either the nature or the frequency of such encounters is not sufficient in some rural schools.

Another possible explanation entails the predominant oral nature of the Zulu culture where print traditionally played a minor role. Through the widespread use of media such as television, newspapers and billboards however, most 'oral' cultures today have had some contact with print, resulting in 'secondary orality' (Ong, 1982). Although most Zulu people therefore are confronted with print in one way or another, there is evidence that the contact has not been great enough to promote a bookish culture among them (Duncan et al., 1973; Solarsh, 2001). The possibility therefore exists that the orality of the culture, albeit secondary orality, plays a role in this phenomenon.

The same argument could be made for the fact that question marks were not interpreted as indicating a question. It is not possible at this stage to determine why participants did not interpret arrows and question marks conventionally, and more research is needed. However, this phenomenon should be kept in mind when a symbol set/system is introduced to this population. Special training in the use of arrows and even conventional literacy symbols might prove beneficial (Moolman \& Alant, 1997).

\section{POSSIBLE INFLUENCES ON RESULTS}

\section{Consistency between sesșions}

In order to ensure that instructions for the test procedure were consistent across sessions, the instructions of 9 out of 13 sessions $(70 \%)$ were played back to one of the translators following data collection.
A checklist comprising all instructions from the test protocol was used to record which instructions were used and which were.left out or modified for each session. Consistency was calculated by dividing the number of instructions used correctly, by the total number of instructions required for each session. An average across sessions was then calculated. Accordingly, consistency across sessions was $94 \%$ (range varied between $80 \%$ and $100 \%)$.

\section{Total frequency of selection of symbols}

The methodology required only one choice per page, to ensure that all 36 symbols were available every time a participant had to make a choice. Consequently the possibility existed for a single symbol to be chosen in response to more than one label or to none. A frequency, procedure showing how often each participant chose each symbol was performed on the data. The procedure revealed that participants commonly selected certain symbols two or three times, and that in one case a symbol was selected up to nine times. It was hypothesised that the more a symbol was selected, the higher the frequency of correct responses would be. To test this hypothesis, the frequency of correct responses per symbol and the total frequency of selection per symbol were plotted on the same chart and compared (Figure I).

It is evident that there is no relationship between the two sets of data to support the hypothesis. It can be concluded that the frequency of correct responses was not a function of total frequency of selection of symbols.

\section{Position of symbols on communication overlay}

The possibility exists that participants were influenced in their choosing of symbols by factors such as placement on the periphery or in the centre of the overlay. It was hypothesised that symbols on the periphery could draw more attention because of less 
competing stimuli surrounding them. Conversely it could be supposed that the four symbols in the centre of the overlay would draw more attention because viewers focus there first. A further possibility would be for participants to concentrate on the symbols in the top left quadrant of the overlay. Fonseca and Lassey $(1964$, in Duncan et al., 1973) found that literate individuals preferred the top left quadrant of a page. A qualitative analysis was performed in order to reveal possible patterns visually. The analysis entailed shading the blocks of a matrix-36 with differing intensities to indicate higher and lower frequencies of selection as a function of position on the overlay. This was done once for the total frequency of selection and then again for frequency of correct responses. These results were then compared. The analysis revealed that symbols placed on the periphery, the centre and the top left quadrant of the overlay were not selected more often in total, nor more often in response to the correct label. It seems that physical placement did not influence selection.

Another factor related to position could have influenced the choices of participants. To facilitate efficiency; the symbols on various communication overlays are consistently grouped according to grammatical categories namely Social (pronouns, whwords, exclamation words and negative words), Verbs, Descriptors (adjectives and adverbs), Prepositions and Nouns (Goossens', Crain \& Elder, 1992). The key concept of each symbol's label serves as a basis for dividing the symbols into these categories. Symbols belonging to the same category are placed together so that they can be colour coded for easy access. Each overlay is therefore roughly divided into five columns, with all Social symbols placed to the very left of the overlay, followed by Verbs, Descriptors, Prepositions, and Nouns to the very right of the overlay. An informal quantitative analysis was performed in order to determine which of the categories was the most iconic.

Although verbs were chosen most often on average, nouns were more often identified correctly, revealing that nouns were the most iconic symbols on the display. These results seem to confirm that of Mizuko (1987) and Bloomberg, Karlan and Lloyd (1990).

\section{Gender}

A recent study in iwhich the target population overlapped with that of the present study, revealed significant gender differences in thinking skills (Solarsh, 2001). Conversely, Duncan et al. (1973) found that the rural Zulu group they studied, performed: poorly on several measures of pictorial perception, regardless of gender, whereas results from rural Tsonga, urban Tsonga and urban Zulu groups did reveal significant gender differences. They hypothesised that in both Tsonga groups and the urban Zulu group, boys performed better than girls since it was more common for boys than for girls to attend school. However, very few children from the rural Zulu group attended school leading to minimal exposure to western pictorial conventions for both genders, thus both genders performed poorly.

The data of the present study was subjected to a chi square test in order to compare the number of correct responses given by boys and girls for every symbol. A significant difference was revealed for Symbol 11 only, $\chi^{2}(1, N=94)=9.7339, p=0.0018$. It seems that gender did not influence the accuracy of participants in identifying PCS symbols. These results agree with the findings of Duncan et al. (1973), although a different explanation for the absence of gender differences may be offered. Today it is equally likely for children from both genders to attend school. The fact that both genders generally have equal opportunities for exposure to pictures and symbols possibly explains the lack of difference in performance.

\section{CONCLUSION}

The iconicity of the selected PCS symbols was generally low for the population studied. This finding serves as a reminder that although PCS had been described as one of the most iconic symbol sets (Mirenda \& Locke, 1989; Mizuko, 1987), the meanings of these symbols are still not entirely guessable for the population studied. A factor that could have contributed to low iconicity in this population was the presence of arrows in many of the symbols. It might prove profitable to use a symbol set/system that employs more postural cues and fewer arrows. Alternatively, clinicians must be aware that special training in the use of arrows might be needed. In future, investigation should be made into how rural Zulu mother-tongue speakers interpret arrows and why.

Furthermore, the unmodified use of commercially available communication overlays containing PCS symbols clearly is not ideal in the South African context. Many* of the themes of the overlays and the concepts dépicted on them do not promote experiential equivalence with Southern African cultures. It is suggested that clinicians choose themes that are relevant to their clients, and then compile communication overlays relating to those themes and the experiential background of the client.

It has been mentioned that the presentation of an array of symbols all related to the same theme, might have had an influence on iconicity and distinctiveness values. Yet symbols are most often used in such a context. It is therefore argued that whatever influence these factors had on the values obtained, the influence served to make the values more functional and socially valid. It is suggested. that this methodology be considered in future iconicity studies.

The construct of "distinctiveness" could hold promise for the field of AAC. It should be validated and its influence on the learnability of symbols investigated. Furthermore, children's perceptions of indicators like arrows need to be explored in more depth.

This study should be seen as a 'first step' towards understanding iconicity in the context of a specific culture, and it clearly shows the need for such research. It is hoped that it will be instrumental in motivating others to investigate the iconicity of graphic representational systems for South African cultures before headlong implementation.

\section{ACKNOWLEDGEMENTS}

This article is the culmination of a thesis submitted by the first author, in partial fulfillment of the requirements of the Masters degree in Augmentative and Alternative Communication, under supervision of the 
second author. The financial assistance of the National Research Foundation (NRF) towards this research is hereby acknowledged. Opinions expressed and conclusions arrived at, are those of the author and are not necessarily to be attributed to the National Research Foundation.

\section{REFERENCES}

Blischak, D. M., Lloyd, L. L., \& Fuller, D. R. (1997) Terminology issues. In L. L. Lloyd, D. R. Fuller \& H. H. Arvidson (Eds.), Augmentative and alternative communication: $A$ handbook of principles and practices (pp. 38-42). Boston: Aliyn \& Bacon.

Bloomberg, K., Karlan, G. R., \& Lloyd, L. L. (1990). The comparative translucency of initial lexical items represented in five graphic symbol systems and sets. Journal of Speech and Hearing Research, 33, 717-725.

Bracken, B. A., \& Barona, A. (1991). State of the art procedures for translating, validating and using psycho-educational tests in cross-cultural assessment:; School Psychology International, 12, 119-132.

Brislin, R. W. (1980). Translation and content analysis of oral and written materials. In H. C. Triandis \& J. W. Berry (Eds.), Handbook. of crosscultural psychology: Vol. 2. Methodology (pp. 389-444). Boston: Allyn \& Bacon.

Burger, D. (Ed.). (2000). South Africa Yearbook 2000/1. Retrieved April 17, 2001, from http://www.gov.za/yearbook/rainbow.htm.

DeLoache, J. (1991). Symbolic functioning in very young children: Understanding of pictures and models. Child Development, 62, 736-752.

Deregowski, J. B. (1980a). Illusions, patterns and pictures. London: Academic Press.

Deregowski, J. B. (1980b). Perception. In H. C. Triandes \& W. Lonner (Eds.), Handbook of cross-cultural psychology: Vol. 3: Basic processes (pp. 21-115). Boston: Allyn \& Bacon.

Doherty, J. E., Daniloff, J. K., \& Lloyd, L. L. (1985). The effect of categorical presentation on AmerInd transparency. Augmentative and Alternative Communication, 1, 10-16.

Duncan, H. F., Gourlay, N., \& Hudson, W. (1973). A study of pictorial perception among Bantu and white primary school children in South Africa. (Human Sciences Research Council Publication Series no. 31). Johannesburg, South Africa: Witwatersrand University Press.

Fuller, D. R. (1987). Effects of translucency and complexity on the associative learning of Blissymbols by cognitively normal children and adults. (Doctoral dissertation, Purdue University, 1987). Dissertation Abstracts International, 49, 710B.

Fuller, D. R. (1997). Initial study into the effects of translucency and complexity on the learning of Blissymbols by children and adults with normal cognitive abilities. Augmentative and Alternative Communication, 13, 30-39.

Fuller, D. R., Lloyd, L. L., \& Stratton, M. M. (1997). Aided AAC symbols. In L. L. Lloyd, D. R.
Fuller \& H. H. Arvidson (Eds.), Augmentative and alternative communication: $A$ handbook of principles and practices (pp. 48-79). Boston: Allyn \& Bacon.

Goossens', C. A., Crain, S., \& Elder, P. (1992). Engineering the preschool environment for interactive, symbolic communication. Birmingham, AL: Southeast Augmentative Communication Publications.

Goossens', C. A., Crain, S., \& Elder, P. (1996). Communication displays for engineered preschool environments: Book H. Matrix-32 and matrix-36 displays. Solana Beach, CA: MayerJohnson Co.

Haupt, E. (2001). The iconicity of selected Picture Communication Systems for rural Zulu-speaking children. Unpublished master's thesis, University of Pretoria, South Africa.

Hoemann, H. W. (1975). The transparency of meaning of sign language gestures. Sign Language Studies, 7, 151-161.

Johnson, R. (1981). The picture communication symbols. Solana Beach, CA: Mayer-Johnson Co.

Johnson, R. (1985). The picture communication symbols: Book II. Solana Beach, CA: MayerJohnson Co.

Johnson, R. (1992). The picture communication symbols: Book III. Solana Beach, CA: MayerJohnson Co.

Lloyd, L. L., \& Fuller, D. R. (1990). The role of iconicity in augmentative and alternative communication symbol learning. In W. I. Fraser (Ed.), Key issies in mental retardation research (pp. 295-306). London: Routledge.

Lloyd, L. L., Fuller, D. R., \& Arvidson, H. H. (1997). Augmentative and alternative communication: $A$ handbook of principles and practices. Boston: Allyn \& Bacon.

Lloyd, L. L., Loeding, B., \& Doherty, J. E. (1985). Role of iconicity in sign language acquisition: A response to Orlansky and Bonvillian (1984). Journal of Speech and Hearing Disorders, 50, 299-301.

Luftig, R. L. (1983). Variables influencing the learnability of individual signs and sign lexicons: A review. Journal of Psycholinguistic Research, $12,361-376$

Luftig, R. L., Page, J. L., \& Lloyd, L. L. (1983). Ratings of translucency in manual signs as a predictor of sign learnability. Journal of Childhood Communication Disorders, 6, 117134.

Mirenda, P., \& Locke, P. A. (1989). A comparison of symbol transparency in nonspeaking persons with intellectual disabilities. Journal of Speech and Hearing Disorders, 54, 131-140.

Mizuko, M. (1987). Transparency and ease of learning of symbols represented by Blissymbols, PCS, and picsyms. Augmentative and Alternative Communication, 3, 129-136.

Moolman, E., \& Alant, E. (1997). The teaching of Blissymbols as a bridge into literacy for children with cognitive impairments: A comparison of two training, approaches. The South African Journal of Communication Disorders, 44, 73-86. 
Musselwhite, C. R., \& Ruscello, D. M. (1984). Transparency of three symbol communication systems. Journal of Speech and Hearing Research, 27, 436-443.

Ong, W. J. (1982). Orality, and Literacy. London: Routledge.

Retief, A. (1988). Method and theory in cross-cultural psychological assessment. (Investigation into research methodology: Research report nr. 6). Pretoria, South Africa: Human Sciences Research Council.

Schneider, M., Claassens, M., Kimmie, Z., Morgan, R., Naicker, S., Roberts, A., \& McLaren, P. (1999). The extent of moderate and severe reported disability and the nature of the disability experience in South Africa. Pretoria, South Africa: Community Agency for Social Enquiry.

Solarsh, B. (2001). Verbal solutions of rural Zuluspeaking children to problems encountered in everyday life. Unpublished doctoral dissertation, University of Pretoria, South Africa.

Taylor, O. L., \& Clarke, M. G. (1994). Culture and communication disorders: A theoretical framework. Seminars in Speech and Language, $15,103-114$

Witkin, H. A. (1967). A cognitive styles approach to cross cultural research. International Journal of Psychology, 2(4), 233-250. 


\section{INFORMATION FOR CONTRIBUTORS}

\section{NATURE OF PUBLICATION}

The South African Journal of Communication Disorders publishes reports and papers concerned with research, and critically evaluative theoretical and philosophical conceptual issues dealing with aspects of human communication and its disorders, service provision, training and policy.

The South African. Journal of Communication Disorders will not accept material which has been published elsewhere or that is currently under review by other publications.

\section{MANUSCRIPT STYLE AND REQUIREMENTS}

- Articles must he accompanied by a covering letter providing the author's address, telephone and fax numbers and e- mail address.

- Articles must be on A4 pages in double spacing and in a font size of 12

- Three print outs of the article must be submitted.

- ONE exact copy of the article on disk must be submitted. Filenames must include the first author's initials and a clearly identifiable key word and must he type-written on the last line of the last page of the Reference list (for retrieval purposes only).

- $\quad$ Articles must not exceed $\underline{30}$ pages.

- The title page of ONE must contain:

- Title of the article.

- Full names of the authors.

- Institutional affiliation.

- Abstract of the article in the language of the article.

- The title page of the remaining TWO copies must NOT contain the authors names or institutional affiliations.

- Each article must contain an abstract of no more than 200 words.

- All abstracts must be in ENGLISH, irrespective of the language in which the article was written.

- Each article must provide 5-7 KEY WORDS for indexing purposes.

- All contributions are required to follow strictly, the style specified in the Publication Manual of the American Psychological Association (APA Pub. Man., 2001).

- Headings are NOT NUMBERED. The order of importance is indicated as follows:

- Main heading in capitals and bold print

- Sub-headings in capitals, bold and italic print.

- Sub-subheadings in upper and lower case bold and italic print.

- Sub-sub-sub-heading in upper and lower case hold print.

- Major headings, where applicable, must be in the order of INTRODUCTION, METHOD, RESULTS, DISCUSSION, CONCLUSION, ACKNOWLEDGEMENTS, REFERENCES.

- All paragraphs should be indented.

- All tables, figures and illustrations must he numbered - and provided with titles.

- The title of tables, which appear above, and of figures, which appear below, must he concise but explanatory.

- Allow for $50-75 \%$ reduction in printing of tables, figures and illustrations.

- Each table, figure or illustration must appear on a SEPARATE page and be print ready. Preferably NOT printed on colour printers.

- Do not include more than 10 tables, figures or illustrations.

\section{REFERENCES}

- References must be cited in the text by surname of the author and the date, e.g. Van Riper (1971).

- Where there are more than two authors, after the first occurrence, et al. may be used.

- The names of all authors must appear in the Reference List, which must he listed in strict alphabetical order in triple spacing at the end of the article.

- All references must be included in the List, including secondary sources, (APA Pub. Man. 2001).

- Only acceptable abbreviations of journals may be used (see DSI-1 ABSTRACTS, October; or The World List of Scientific Periodicals).

- The number of references should not exceed 30, unless specifically warranted.

\section{EXAMPLES}

Locke, J.L. (1983). Clinical Psychology: The explanation and treatment of speech sound disorders. J. Speech Hear Disord., 48 339-341.

Penrod, J.P. (1985). Speech discrimination testing, In J Katz (Ed.), Handbook of clinical audiology ( $3^{\text {rd }}$ ed.) Baltimore: Williams \& Wilkins.

Davis, G. \& \& Wilcox, M.J. (1985). Adult aphasia rehabilitation: Applied pragmatics. San Diego, CA: CollegeHill.

\section{EDITING}

- Articles must be corrected for grammar and style prior to submission.

- The manuscript style of the article must be strictly according to the guidelines provided.

- Only articles complying with the above requirements will be accepted for review.

\section{REVIEWING SYSTEM}

- The peer review of refereeing system is employed as a method of quality control of this publication.

- Peer reviewers are selected by the editor based on their expertise in the field and each article is sent to two independent reviewers to assess the quality of the manuscript's scientific and technical content.

- The blind peer review system is employed during which the names of the author/authors are not disclosed to the reviewers.

- The editor retains the final responsibility for decisions regarding revision, acceptance or rejection of the manuscript.

DEADLINE FOR CONTRIBUTIONS. $30^{\text {th }}$ January each year

QUERIES, CORRESPONDENCE \& MANUSCRIPTS: should he addressed to The Editor, South African Journal of Communication Disorders, South African SpeechLanguage-Hearing Association, PO Box 5710, THE-REEDS, 0158 , South Africa

\section{Copyright}

The copyright of all articles printed in The South African Journal of Communication Disorders is reserved by The South African Speech-Language-Hearing Association (SASLHA) 